\title{
Influence of electric field on fracture toughness and fatigue crack growth for an actuator piezo-ceramic PIC151
}

\author{
X.-P. Zhang ${ }^{1}$, S. Galea ${ }^{2}$, L. Ye ${ }^{1}$ and Y.-W. Mai ${ }^{1}$ \\ 1. Centre of Expertise in Damage Mechanics, School of Aerospace, Mechanical and Mechatronic \\ Engineering, the University of Sydney, NSW 2006, Australia \\ 2. Air Vehicles Division, Platform Sciences Laboratory, DSTO, Melbourne, Australia
}

\begin{abstract}
Experimental investigations were carried out to characterize fracture and fatigue behaviours of an actuator piezoelectric ceramic under the combined loading of a high electric field and a mechanical stress. Results show that there exists a strong anisotropic effect on fracture toughness and electric field induced fatigue crack growth in polarized PZT. It is found that the surface fracture toughness in the orien -tation parallel to the polarization direction is much higher than that in the transverse orientation. Under a positive electric field, increasing electric field intensity reduces the fracture toughness in the transverse orientation but enhances that in the parallel orientation. However, the reverse is true under a negative electric field. Low electric field intensity does not result in fatigue crack growth in PZT. For a relatively high applied electric field, the cracks initially grow fast and then are arrested. This result is very significant for the long-term durability of PZT actuators.
\end{abstract}

Keywords: piezo-ceramic, fracture toughness, electric field induced fatigue crack growth, anisotropic effect

\section{INTRODUCTION}

In recent years, there have been many interests to build 'smart' structures, microelectronics and microelectromechanical systems utilising the special functionality of piezoelectric materials as sensors, actuators, controllers and so forth, for the purposes of controlling structural shape and vibration and suppressing noise. As is widely known, in the above structures or systems, piezoelectric materials are subjected to very complicated service conditions of mutual electric and mechanical stress fields. Thus, the electro-mechanical properties of piezoelectric materials, and particularly their durability and reliability, are critical to help realise the functions required. However, the integrity and failure characteristics and the degra -dation of piezoelectric materials are less well understood. Especially, the anisotropy of fracture toughness and electric field induced fatigue crack growth behaviours in polarized piezoceramics have long been recognised to affect significantly the service performance of actuators and sensors. In the past two decades, many theoretical studies including finite element modelling have been conducted to characterise the fracture and damage of piezoelectric ceramics under electrical, mechanical or combined electro-mechanical loading modes [1-6]. Only limited experimental work had been performed to characterize the fracture toughness of polarized piezoelectric materials under an applied electric field [7-12]; especially there is still a lack of basic understanding of the cyclic electric field induced fatigue crack growth behaviour.

In the present study, the influence of applied electric field on surface fracture toughness and the alternating electric field induced fatigue crack growth behaviours for polarized PZT pellets (PIC 151) were characterized, and the damage behaviour of PZT bonded on a structure surface under the combined loading of a high electric field and a mechanical stress was investigated.

\section{EXPERIMENTAL PROCEDURE}

\subsection{Piezoelectric material}

The piezoelectric ceramic used was PIC 151, produced by Physik Instrumente (PI GmbH \& Co., Germany), which could be compared to PZT-5H types in terms of electromechanical and dielectric parameters, etc, and was used in many applications to replace PZT-5H. The major physical and mechanical properties of PIC 151 are: density $\rho=7.80 \mathrm{~g} / \mathrm{cm}^{3}$, Curie temperature $=250{ }^{\circ} \mathrm{C}$, modulus, $\mathrm{Y}_{33}=5.26 \times 10^{10} \mathrm{~N} / \mathrm{m}^{2}$, maximum field in poling direction: $2000 \mathrm{~V} / \mathrm{mm}$, coercive field: $\sim 1000 \mathrm{~V} / \mathrm{mm}$, dielectric constants: $\varepsilon_{33}^{T} / \varepsilon_{0}=2100$ and $\varepsilon_{11}^{T} / \varepsilon_{0}=1980$, dielectric loss $(\tan \delta)=15 \times 10^{-3}$, coupling factors: $\mathrm{k}_{\mathrm{p}}=0.62, \mathrm{k}_{33}=0.69$ and $\mathrm{k}_{31}=0.34$, elastic constants: $S_{11}^{E}=66.7 \mathrm{GPa}, S_{33}^{E}=$ $86.2 \mathrm{GPa}$.

\subsection{Fracture toughness estimation subjected to an ap- plied electric field}

Fracture toughness of PZT was determined by the Vickers indentation method [13-18]. As the indentation crack was small compared to the specimen dimensions, an attempt was made to relate the Vickers indentation with analytical solutions for a center-cracked infinite plate with far-field applied loads. For isotropic, homogenous materials, explicit equations for the radial crack evolution by a sharp indenter have been given. For a sufficiently well-defined crack, i.e. $a \gg \mathrm{D}$, where $a$ and D 
are, respectively, measured half-crack length and half diagonal length of the indentation pyramid (see Fig. 1), the stress intensity factor $\mathrm{K}$ can be expressed in terms of material constants, indentation load and indentation crack length as follows [14, 18]:

$$
\begin{aligned}
& K=0.016\left(\frac{E}{H}\right)^{\frac{1}{2}} P a^{-\frac{3}{2}} \\
& H=\frac{P \sin \left(\frac{\alpha}{2}\right)}{2 D^{2}}
\end{aligned}
$$

where $\mathrm{P}$ is applied indentation load in Newton, $\mathrm{E}$ and $\mathrm{H}$ represent Young's modulus and Vickers hardness, respectively (expressed in $\mathrm{N} / \mathrm{m}^{2}$ ). $\alpha$ is apex angle of the Vickers diamond indenter and is normally $136^{\circ}$.

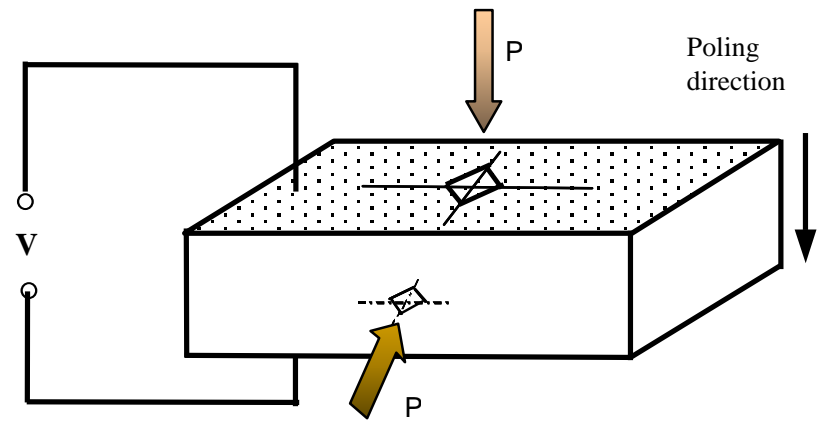

(a) Indentation under an applied electric field.

Vickers

Indenter

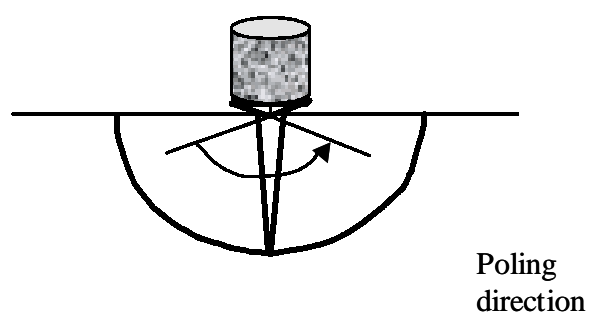

2D

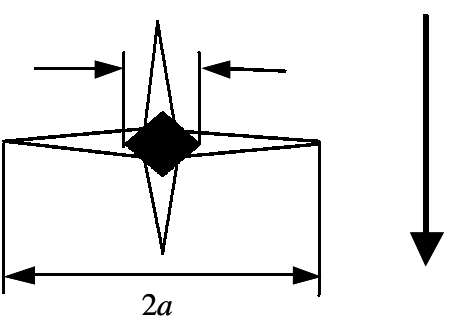

(b) Dimensions of Vickers indentation crack.

Fig. 1. Schematic showing measurement of fracture toughness by Vickers indentation test.
To study the influence of electric polarisation and electric field intensity on fracture toughness and crack growth, Vickers indentations (with a load of $9.8 \mathrm{~N}$ ) were performed under an on-site applied electric field with different intensities up to the coercive field intensity $(\sim 1000$ $\mathrm{V} / \mathrm{mm}$ ). As shown in Fig. 1, Vickers indentations were made on both flat surface and edge of PIC 151 (pellet size is $25 \times 3 \times 2 \mathrm{~mm}$ ). The electric field was applied by a high-voltage Supply/Amplifier/Controller (Model 6120 D, TREK Inc., USA), which could provide an output voltage range of 0 to $\pm 10 \mathrm{kV}$. To prevent electric discharge through air, the measurements were conducted in a small silicon oil tub. Five indentations were conducted at each of field intensities ranging from negative values of $-50 \%$ $\mathrm{E}_{\mathrm{c}},-20 \% \mathrm{E}_{\mathrm{c}}$ to zero electric field and then positive values of $20 \%, 50 \%$ and $100 \% \mathrm{E}_{\mathrm{c}}$.

\subsection{Cyclic electric field induced fatigue crack growth tests in polarized PZT}

To characterize electric field induced fatigue crack growth, an alternating electric field was applied on PIC 151, Fig.1. The experimental system consists of HP VXI mainframe $\left(\right.$ Agilent $^{\odot}$ ), voltage/power amplifier, PC (as a control platform) and a specimen clamping/insolation device etc. The HP VXI mainframe consists of Signal Excitation Unit (SEU), Signal Acquisition Unit (SAU) and Central Control Unit (CCU), etc. The alternating electric field signal (voltage), simulated by Agilent ${ }^{\odot}$ E1441 on the NI Labview ${ }^{\odot}$ platform, was applied to the PZT specimens through SEU after being amplified to a certain voltage level by a PZT amplifier (PiezoSys ${ }^{\odot}$ EPA-104). As the core of the system, CCU performed both as controller and interpreter, supported by the NI Labview ${ }^{\odot}$ platform, and experimental parameters could be imputed and controlled conveniently. Tests were performed in open-air condition. The supply of the electric field was stopped intermittently after a certain period (that is, number of electric field cycles) to conduct measurements. Fatigue crack growth was observed and measured by an optical microscope and a digital camera.

\section{RESULTS AND DISCUSSIONS}

\subsection{Influence of electric field intensity on fracture toughness of polarized PZT \\ (1)Fracture toughness of polarized PZT without an ap- plied electric field}

Figures 2 and 3 show, respectively, Vickers indentations on the surface and edge of a polarized PIC 151 pellet. In Fig.2, cracks in both horizontal and vertical directions have similar lengths, indicating that there is no anisotropy of the surface fracture toughness which is estimated to be $\sim 1.41 \mathrm{MPa} . \mathrm{m}^{1 / 2}$. In Fig. 3, there are two predominant cracks in the direction transverse to the poling direction on the edge of PIC 151. Obviously, there is a strong anisotropy of fracture toughness in the thickness direction, and the average values in these two orientations are: 0.60 (transverse) and 1.49 (parallel) $\mathrm{MPa} . \mathrm{m}^{1 / 2}$, respectively. 


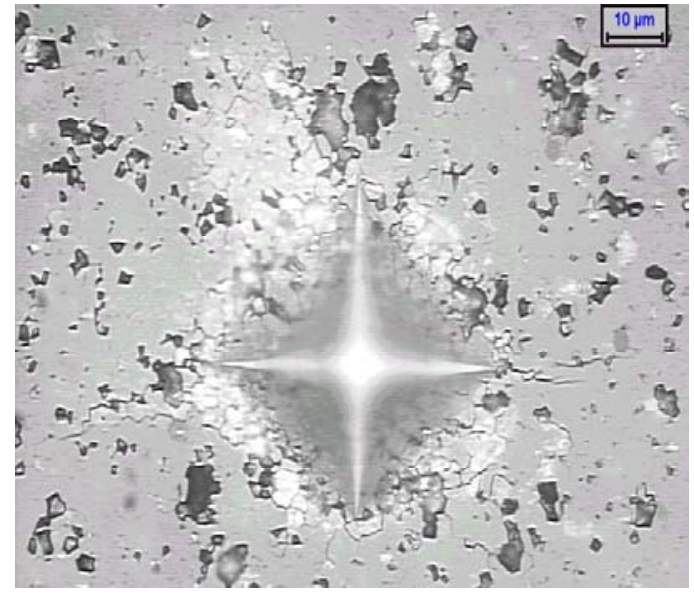

Fig. 2. Vickers indentation on the flat surface of PIC 151.

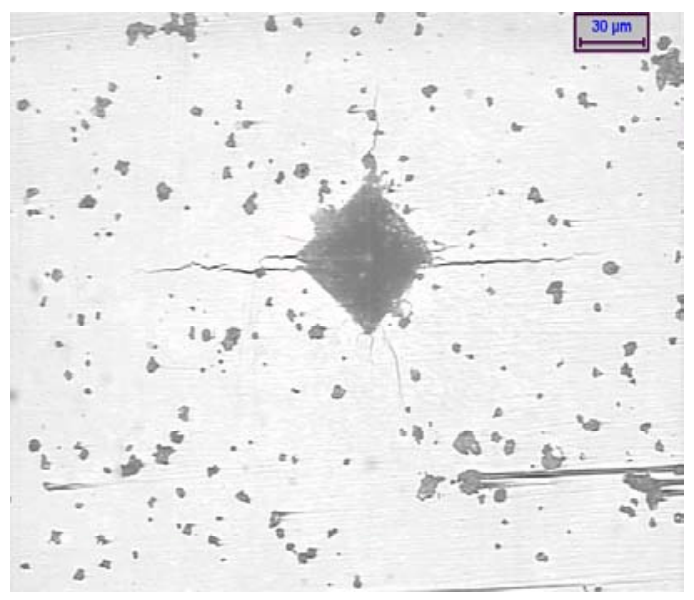

Fig. 3. Vickers indentation on the edge of PIC 151. (Poling direction is vertical)

\section{(2)Influence of electric field on surface fracture tough-} ness of polarized PZT

Results of the effect of applied electric field intensity on indentation crack length and fracture toughness are shown in Figs. 4 and 5, respectively. As manifested in Fig. 4 and also shown in Fig. 3, it is clear that the cracks transverse to poling direction are larger and longer than those in the parallel direction under all electric field intensities. Thus, fracture toughness is much lower in the transverse than it is in the parallel direction. It is noted that under a positive electric field, with increasing electric field intensity, the crack length increases in the transverse orientation but decreases in parallel orientation. Under a negative electric field, the trends are reversed. Hence, the fracture toughness in the transverse direction is much lower than that in the parallel direction in all electric field intensities.

Therefore, there is a strong anisotropic effect on the fracture toughness of polarized PIC 151, and the fracture toughness in the orientation parallel to the polarization direction is $2 \sim 3$ times higher than that in the transverse direction.

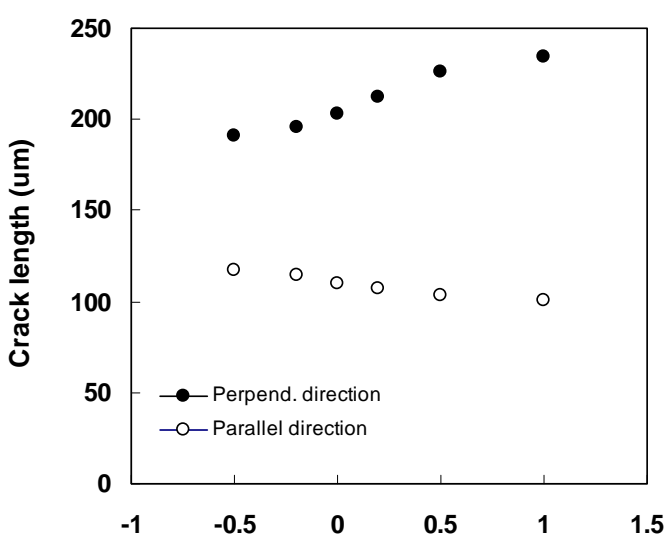

Electric field intensity (E/Ec)

Fig. 4. Influence of electric field on crack length.

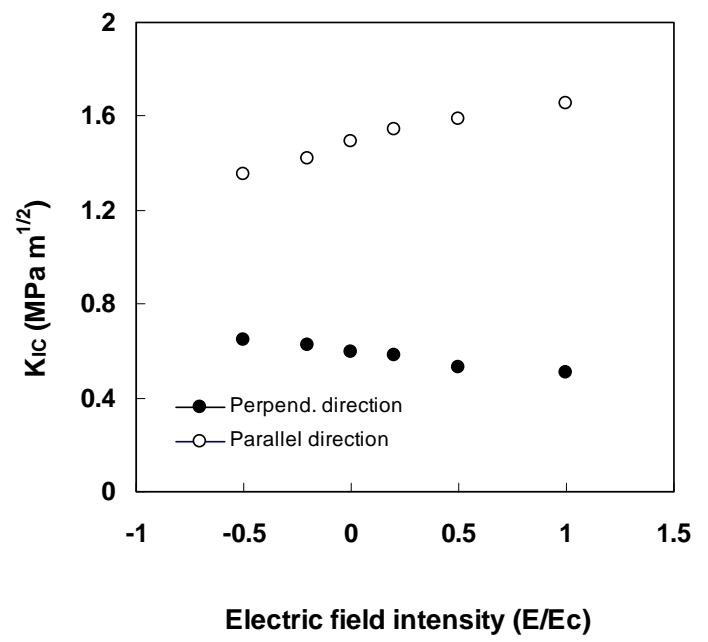

Fig. 5. Influence of electric field intensity on Vickers indentation fracture toughness.

The anisotropic fracture toughness of polarized PZT is mainly due to its domain orientations. The fracture toughness is higher when domain orientation is parallel to the crack growth direction. In contrast, fracture toughness is lower when domain orientation is transverse to crack growth direction. These results are consistent with published data [17, 19]. This observation can be explained by the domain-switching phenomenon that occurs at the crack-tip region due to the tensile stress field [2]. If domain orientation is coincident with crack growth direction, $90^{\circ}$ domain switching occurs at the crack-tip zone causing local stress relaxation and blocking crack growth; thus the fracture toughness becomes higher. When domain orientation is transverse to the crack growth direction, no obvious $90^{\circ}$ domain switching happens, hence no blocking effect on crack growth and the fracture toughness is lower. Theoretical modeling shows similar results [20-21]. 


\subsection{Cyclic electric field induced fatigue crack growth in polarized PZT}

For polarized PIC151 with a Vickers indentation at the center of the surface (the indentation is isotropic, same as Fig.2), after an alternating electric field was applied, with sine waveform and peak-to-peak intensities of $30 \% \mathrm{E}_{\mathrm{c}} \sim$ $85 \% \mathrm{E}_{\mathrm{c}}$, and frequency ranging $50 \sim 200 \mathrm{~Hz}$, on the pellet for $60 \mathrm{~h}$ (i.e. $10.8 \sim 40.3 \times 10^{6}$ electric cycles), no crack growth at the four tips of the indentation could be seen. This implies that for PIC 151, which is free of constraints (that is, not bonded on a structural surface), alternating electric field intensity as high as $850 \mathrm{~V} / \mathrm{mm}$ could not cause any growth of the surface microcracks.

For microcracks on the edge of the same PIC 151, Fig. 6 , observations and measurements showed that at frequen -cies of 50 to $200 \mathrm{~Hz}$ and electric field intensities of $30 \%$ $\sim 50 \% \mathrm{E}_{\mathrm{c}}$, no obvious crack growth was found. However, under an alternating electric field intensity $85 \% \mathrm{E}_{\mathrm{c}}$, electric field induced fatigue crack growth occurred as shown in Figs. 7 and 8. The crack length and extension in the orientation transverse to poling direction versus electric cycle are given in Figs. 9 and 10, respectively. Crack growth in the parallel orientation was not found in the present experiments.

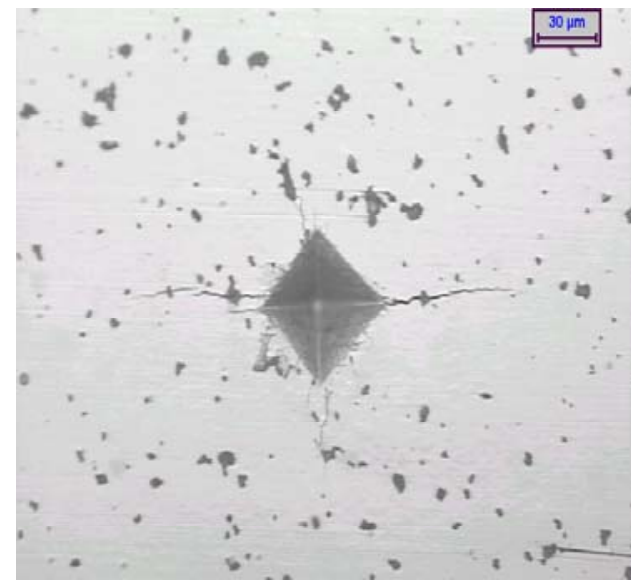

Fig. 6. View of the initial indentation (poling direction is along the vertical direction).

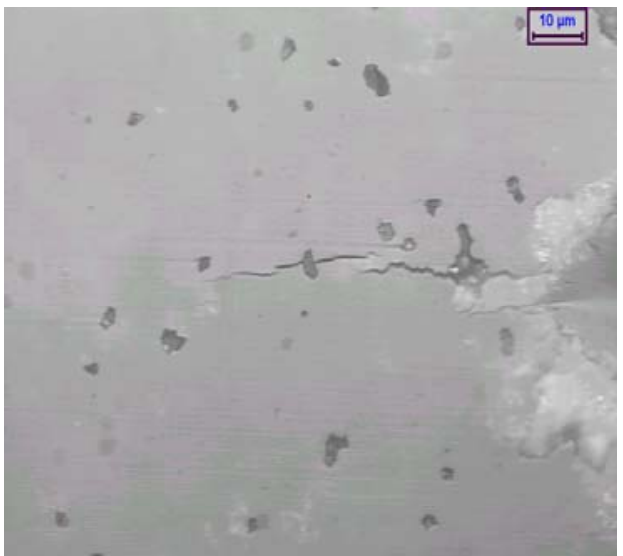

(a) Left side crack in Fig. 6

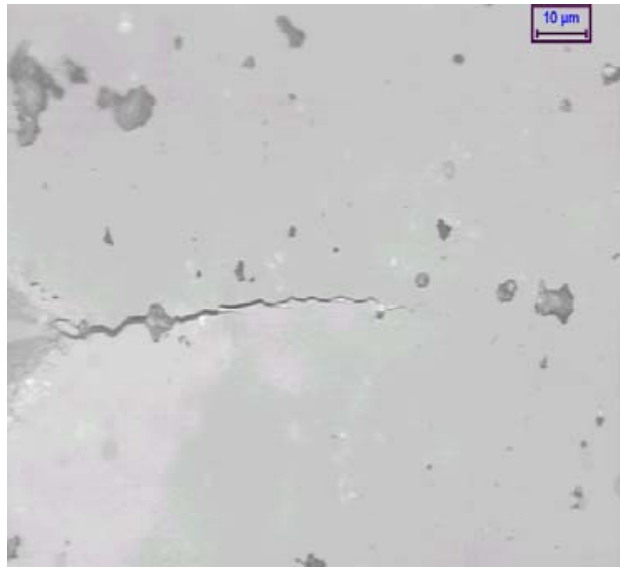

(b) Right side crack in Fig. 6

Fig. 7. Crack growth transverse to poling direction after 150000 electric cycles.

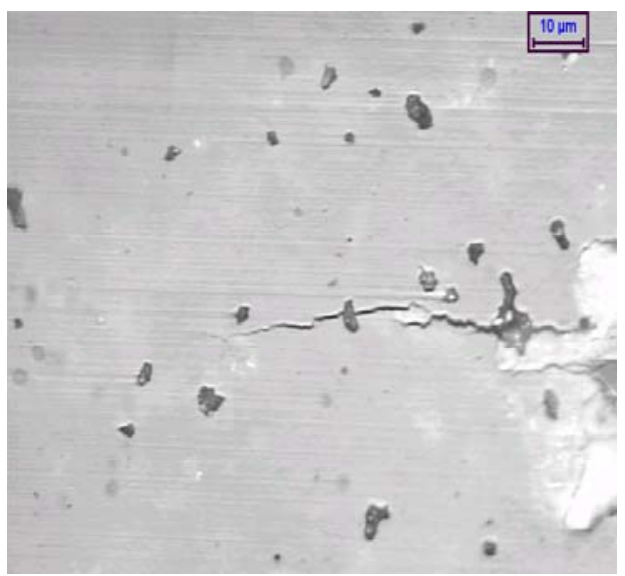

(a) Left side crack in Fig. 6

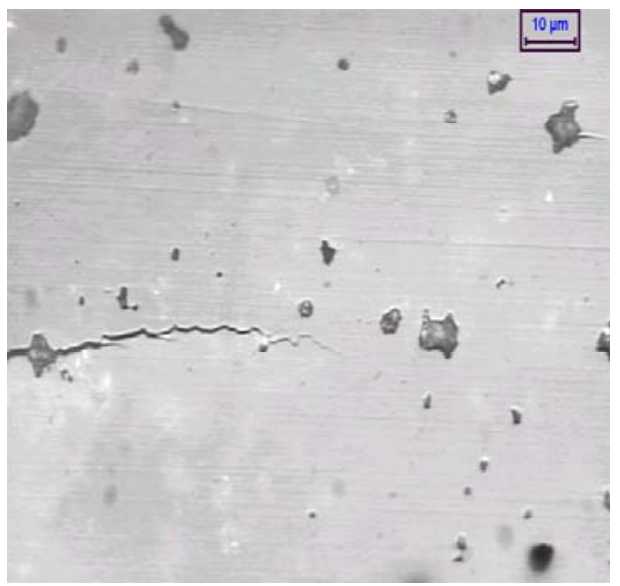

(b) Right side crack in Fig. 6

Fig. 8. Crack growth transverse to poling direction after 300000 electric cycles. 


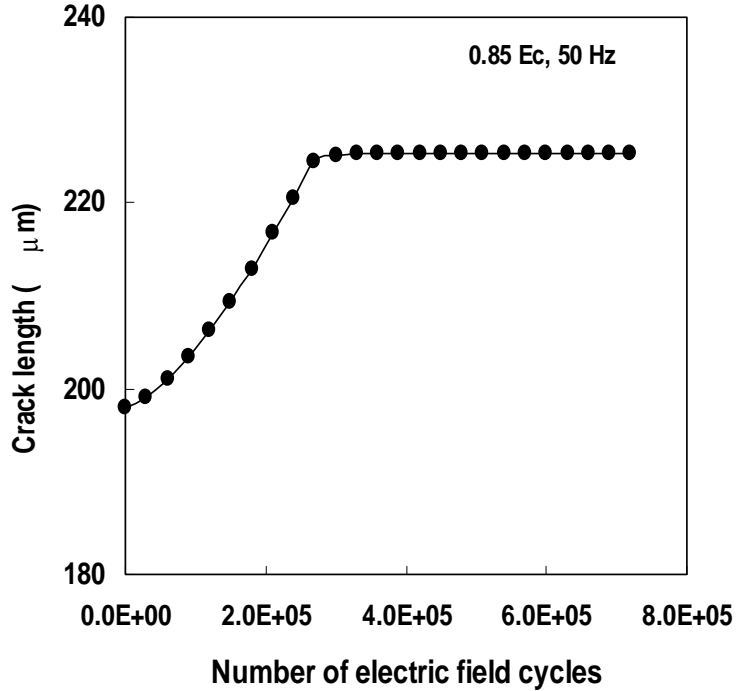

Fig. 9. Crack length versus electric filed cycle number.

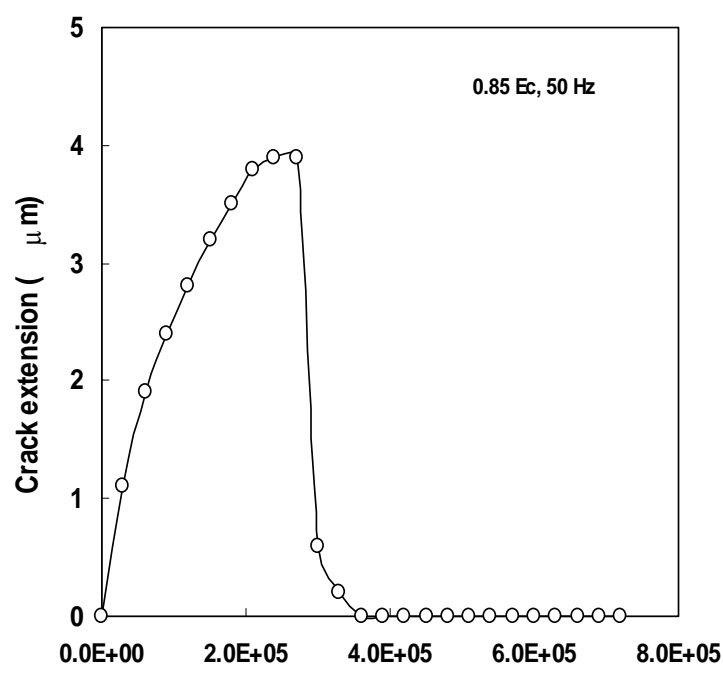

Number of electric field cycles

Fig. 10. Crack extension vs elapsed electric field cycles.

In Figs. 9 and 10, it can be seen clearly that under an electric field intensity $85 \% \mathrm{E}_{\mathrm{c}}$ the crack grew very fast at the initial stage due to the effect of residual stress at the crack-tip, and then slowed down gradually, and finally became non-propagating after the number of electric field cycles exceeded 300000 . To quantify these results, crack propagation rates are plotted against elapsed electric cycles as shown in Fig. 11, where it is clear that the crack had the maximum growth rate at the beginning, and slowed down till it became dormant. In general, the crack growth rate was very small, in the order of $10^{-11} \mathrm{~m} /$ cycle.

In addition, the fracture toughness was also plotted against the elapsed electric field cycles in Fig. 12, in which it decreases gradually to a constant value at about 300000 electric cycles.

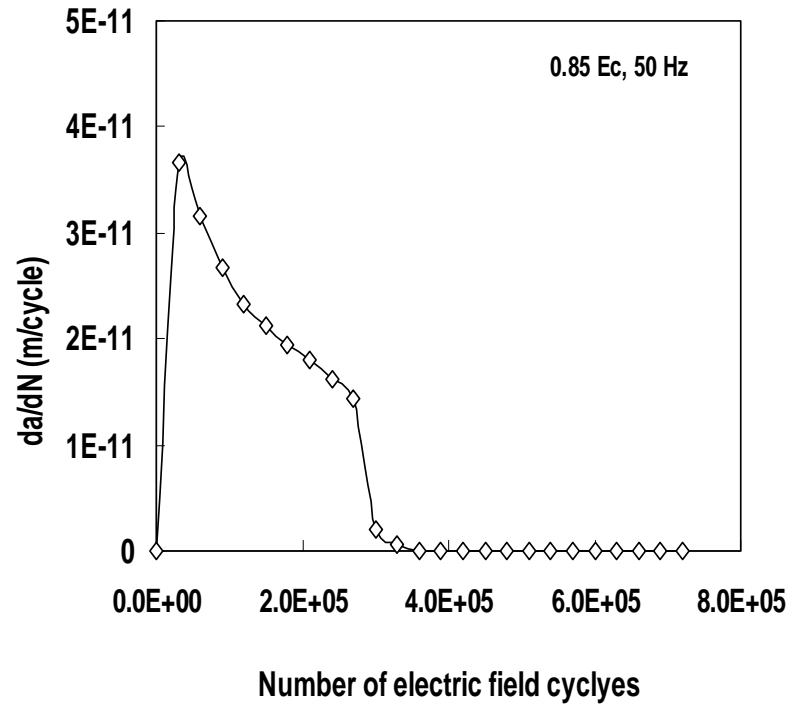

Fig. 11. Crack growth rate $v s$ elapsed electric field cycles.

It should be pointed out that there is no electric induced fatigue crack growth if the alternating electric field intensity is not high, such as $30 \% \sim 50 \% \mathrm{E}_{\mathrm{c}}$. And for a relatively high electric field, for example $85 \% \mathrm{E}_{\mathrm{c}}$ for PIC 151 , cracks initially grow fast and then become arrested. This result is important for the long-term reliability and durability performance of PZT materials as actuators. In this work, further higher alternating electric field intensities, for example above $E_{c}$, were not investigated due to limitations of the facilities used. However, research carried out by Weitzing and Schneider et al [22] on cyclic electric field induced fatigue crack growth behaviour for three different PZT ceramics showed that a cyclic electric field with an intensity as high as 1.5 times $\mathrm{E}_{\mathrm{c}}$ may drive the crack to grow as long as $1 \sim 2 \mathrm{~mm}$, and finally the crack would stop.

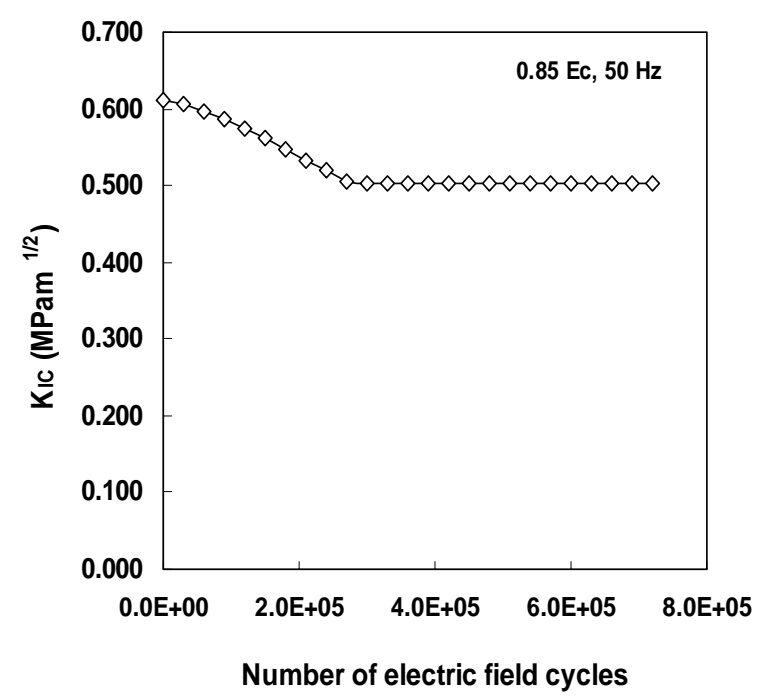

Fig. 12. Fracture toughness vs elapsed electric field cycle. 


\section{CONCLUSIONS}

(a) There is a strong anisotropic effect on the fracture toughness in the thickness direction of polarised PIC 151 PZT. The fracture toughness in the orientation parallel to the polarization direction is much higher than that in the transverse direction.

(b) Electric field has a strong effect on the fracture toughness of monolithic PZT. Under a positive electric field, increasing the electric field intensity causes the fracture toughness to decrease in the transverse orientation but increase in the parallel orientation. These trends are reverse under an applied negative electric field.

(c) Low electric field intensity does not promote fatigue crack growth in polarized PZT. For a relatively high electric field (that is, $85 \% \mathrm{E}_{\mathrm{c}}$ for PIC 151), the crack will grow at a fast rate initially but then becomes arrested after a large number of electrical cycles. This result is significant in the design of long-term reliability and durability of PZT materials as actuators.

ACKNOWLEDGEMENT - This work was supported by AVD, PSL of DSTO (Melbourne, Australia), via a contract to the Centre of Expertise in Damage Mechanics (COE-DM), School of Aerospace, Mechanical and Mechatronic Engineering at the University of Sydney.

\section{REFERENCES}

1. Z. Suo, Smart Structures and Materials, American Society of Mechanical Engineers, 24 (1991), pp. 1-6.

2. Z. Suo, C.-M. Kuo, D.M. Barnett and J.R. Willis, Journal of the Mechanics and Physics for Solids, 40 (1992), pp. 739-765.

3. H. Sosa, Mechanics of Electromagnetic Materials and Structures, American Society of Mechanical Engineers, 161(1993), pp. 63-75.

4. H.J. Gao, T.Y. Zhang and P. Tong, Journal of the Mechanics and Physics of Solids, 45(1997), pp. 491-510.
5. C.C. Fulton and H.J. Gao, Proceedings of SPIE - The International Society for Optical Engineering, 3323 (1998), pp. 119-127.

6. R.M. McMeeking, Engineering Fracture Mechanics, 64 (1999), pp. 217-244.

7. A.G. Tobin and Y.E. Pak, Proceedings of SPIE - The International Society for Optical Engineering: Smart Structures and Materials, 1916(1993), pp. 78-86.

8. H.C. Cao and A.G. Evans, Journal of the American Ceramic Society, 77 (1994), pp. 1783-1786.

9. S.B. Park and C.T. Sun, International Journal of Fracture, 70 (1995), pp. 203-216.

10.C.S. Lynch, Acta Materialia, 46 (1998), pp. 599-608.

11.G.A Schneider and V. Heyer, Journal of the European Ceramics Society, 19(1999), pp. 1299-1306.

12.A. Kolleck, G.A. Schneider and F.A. Meschke, Acta Materialia, 48(2000), pp. 4099-4113.

13.A.G. Evans and E.A. Charles, Journal of the American Ceramic Society, 59 (1976), pp. 371-372.

14.G.R. Anstis, P. Chantikul and B. Lawn, Journal of the American Ceramic Society, 64 (1981), pp. 533-538.

15.G.R. Anstis, P. Chantikul and B. Lawn, Journal of the American Ceramic Society, 64 (1981), pp. 539-543.

16.K. Okazaki, Ceramic Bulletin, 63 (1984), pp. 11501157.

17.K. Mehta and A.V. Virkar, Journal of the American Ceramic Society, 73(1990), pp. 567-574.

18.C.T. Sun and S.B. Park, Proceedings of SPIE - The International Society for Optical Engineering, Smart Structures and Materials, 2441(1995), pp. 213-222.

19.G.G. Pisarenko, V.M. Chushko and S.P. Kovalev, Journal of the American Ceramic Society, 68 (1985), pp. 259-265.

20.W. Yang and T. Zhu, Fatigue and Fracture of Engineering Materials and Structures, 21(1998), pp. 1361-1369.

21.I. Chang and C.T. Sun, Adaptive Structures and Material System, American Society of Mechanical Engineers, AD, 59 (1999), pp. 77-85.

22.H. Weitzing, G.A. Schneider, J. Steffens, M. Hammer and M.J. Hoffmann, Journal of the European Ceramic Society, 19(1999), pp. 1333-1337. 